Service social

\title{
Des recherches et essais sur l'organisation communautaire contemporaine
}

\section{Yvan Comeau et Myreille St-Onge}

Volume 54, numéro 1, 2008

URI : https://id.erudit.org/iderudit/018339ar

DOI : https://doi.org/10.7202/018339ar

Aller au sommaire du numéro

Éditeur(s)

École de service social de l'Université Laval

ISSN

0037-2633 (imprimé)

1708-1734 (numérique)

Découvrir la revue

Citer cet article

Comeau, Y. \& St-Onge, M. (2008). Des recherches et essais sur l'organisation communautaire contemporaine. Service social, 54(1), 1-5.

https://doi.org/10.7202/018339ar d'utilisation que vous pouvez consulter en ligne.

https://apropos.erudit.org/fr/usagers/politique-dutilisation/ 


\title{
AVANT-PROPOS
}

\section{Des recherches et essais sur l'organisation communautaire contemporaine}

\author{
Yvan COMEAU \\ Professeur titulaire \\ Responsable du numéro spécial \\ École de service social \\ Université Laval \\ Myreille ST-ONGE \\ Professeure agrégée \\ Directrice de la revue Service social \\ École de service social \\ Université Laval
}

En publiant ce numéro spécial sur l'organisation communautaire, la revue Service social souhaite contribuer à faire le point sur cette méthode du travail social. À ce propos, la revue publiait, en 1985, un numéro lui étant entièrement consacré. L'organisation communautaire était alors pratiquée depuis une dizaine d'années au Québec dans les Centres locaux de services communautaires (CLSC). Ce numéro faisait partie, avec quelques ouvrages qui paraissent au même moment (Ampleman et al., 1983, 1987; Lamoureux, Mayer et Panet-Raymond, 1984; Marcotte, 1986; Savaria, 1985), des premières systématisations de l'organisation communautaire québécoise telle qu'on la retrouve au milieu des années 1980.

La décision de produire le présent numéro tient également d'un événement à souligner, soit le $20^{\text {ième }}$ anniversaire du Regroupement québécois des intervenantes et intervenants en action communautaire (RQIIAC) qui changera d'appellation en 2008. Le Regroupement voit le jour en 1988 dans une conjoncture formée de deux éléments. Le premier est le très grand besoin que ressentent alors les organisatrices et organisateurs communautaires d'échanger sur leurs pratiques car, à cette époque, les lieux de formation et d'échange sont pour eux plutôt rares. Le deuxième élément concerne la remise en question que fait le rapport Brunet, publié en 1987, du volet social des CLSC à l'aube d'une réforme majeure du système de santé et des services sociaux que suggère le rapport de la Commission Rochon rendu public en 1988. 
On peut également considérer que la publication de ce numéro coïncide avec la fin d'une époque et, peut-être, l'avenir le dira, le début d'une nouvelle ère pour l'organisation communautaire. Le marqueur historique pourrait bien être, au Québec, la réforme du système de santé et de services sociaux lancée par le projet de loi 25 (Loi sur les agences de développement des réseaux locaux de services de santé et de services sociaux) sanctionné par l'Assemblée nationale, le 18 décembre 2003, suivi par le projet de loi 83 (Loi modifiant la Loi sur les services de santé et les services sociaux) que le Parlement adopte en novembre 2005. Dans la foulée de la loi 25, les Agences ont mis en place un nouveau mode d'organisation des services axé sur des réseaux locaux de services (RLS). En juin 2004, on assiste donc au Québec à la création de 95 réseaux locaux de services dont l'assise est un nouvel établissement, le Centre de santé et de services sociaux (CSSS) qui, dans la plupart des régions, naît de la fusion de CLSC, de centres d'hébergement et de soins de longue durée (CHSLD) et d'un centre hospitalier de soins généraux et spécialisés (CHSGS). Ces mesures modifient considérablement les établissements publics et leur mission et auront vraisemblablement des incidences importantes au Québec sur les professionnels de l'intervention, sur le travail social et sur l'organisation communautaire.

Les textes réunis dans le présent numéro traitent de plusieurs aspects marquant actuellement l'organisation communautaire au Québec. Le premier article signé par l'équipe de Yvan Comeau permet de saisir les transformations de la pratique de l'organisation communautaire dans les réseaux public et communautaire, à partir d'une comparaison des résultats d'une recherche auprès de 419 organisatrices et organisateurs communautaires réalisée en 2004 et d'une autre conduite en 1988 auprès d'organisateurs communautaires œuvrant en CLSC. Le lectorat en apprendra davantage sur ces personnes, sur les établissements qui les embauchent, sur leur pratique et sur les tâches concrètes qu'elles exercent. II serait plus juste d'ajouter que la perspective de l'article est davantage comparative et explicative que proprement descriptive. En effet, les auteurs veulent identifier les phénomènes qui contribuent à modeler l'organisation communautaire et à lui donner les formes qu'elle prend dans le temps et dans les différentes organisations.

Dans le second article, René Lachapelle et Denis Bourque s'intéressent à la pérennité des fondements de l'organisation communautaire dans le contexte du développement accru des interventions en santé publique dans les CLSC maintenant rattachés aux CSSS. II convient de rappeler que les programmes de santé publique ont été renforcés avec le projet de loi 120 (Loi sur les services de santé et les services sociaux) adopté en août 1991 annonçant le début de la réforme Côté. Celle-ci prévoyait notamment le remplacement des 32 départements de santé communautaire par 16 directions régionales de santé publique alors rattachées à autant de Régies régionales de la santé et des services sociaux (RRSSS). Maintenant 18 directions de santé publique (incluant le Nunavik et les Terres-Cries-de-la-Baie-James) sont liées aux 18 Agences de la santé et des services sociaux de la province créées par la loi 25. Les auteurs se demandent dans quelle mesure l'organisation communautaire peut conserver son autonomie dans l'application d'un programme développé par des experts tout en étant respectueuse de la participation des communautés dans sa mise en œuvre. À partir 
d'une recherche fondée sur 18 entrevues réalisées avec des organisatrices et organisateurs communautaires œuvrant dans quatre programmes de santé publique de 10 régions administratives du Québec, les auteurs en arrivent à identifier les conditions permettant de préserver le caractère communautaire à l'intervention. Les auteurs distinguent les deux perspectives de l'approche socio-institutionnelle ou planning social : technocratique et participative. En ce sens, ils convient le lectorat à explorer les facteurs favorables et défavorables à l'exercice d'une approche socioinstitutionnelle participative qui permet la participation des personnes et des communautés directement concernées par les problématiques couvertes par les programmes de santé publique.

Comme d'autres méthodes d'intervention en travail social, l'organisation communautaire se développe dans de nouveaux créneaux. On peut considérer sous cet angle, la contribution de Mathieu Cook sur l'abandon scolaire et celle de Charles Gaucher sur la communauté des Sourds. L'importance qu'a prise la problématique de l'abandon scolaire au cours des dernières années peut s'expliquer par la connaissance accrue de ses incidences sur la pauvreté et sur les dépenses sociales. Comme c'est souvent le cas pour une innovation, l'étude de cas s'avère une démarche intéressante pour rendre compte de l'organisation communautaire dans ce domaine. L'expérience du Conseil régional de prévention de l'abandon scolaire du Saguenay-Lac-Saint-Jean (CRÉPAS) sert ici de prototype. Dans cette expérience, on reconnaît plusieurs traits caractéristiques de l'organisation communautaire: une analyse critique et complexe d'un phénomène, un intérêt pour les groupes défavorisés, l'importance accordée à la prévention, à la concertation du milieu, à la mobilisation des acteurs et au transfert du leadership dans les communautés.

L'article de Charles Gaucher touchant l'organisation communautaire avec la communauté des Sourds permet de soulever un phénomène de société qui interpelle non seulement le service social, mais plus largement les sciences sociales. II s'agit du développement des identités qui est un des traits de la société contemporaine dite « en réseaux » ou « informationnelle capitaliste » (Castells, 2000). Comme il arrive souvent dans plusieurs domaines, l'intervention se déroule avec, en toile de fond, le choc des identités dans lequel est impliquée bien malgré elle la personne qui intervient. Ainsi en est-il du travail d'intellectuel avec la communauté des Sourds. Dans son article, Charles Gaucher soulève cette question complexe et propose d'aborder le problème en décortiquant la notion de communauté. L'auteur montre les différentes significations que prend la notion de communauté (un milieu de vie, un fait associatif, une identité à partir d'un trait définitionnel et d'une communauté englobante ou sociétale) et opte pour une perspective intégratrice de ces variantes. Pour l'auteur, il existe bel et bien une communauté sourde que le langage des signes notamment a contribué à cimenter. Or, comme c'est le cas pour l'intervention et la recherche avec toute communauté différente de la sienne, les rapports comportent des paradoxes. D'emblée, il n'est pas assuré que l'intervention puisse être à la fois respectueuse des différences et capable de prendre en compte les enjeux sociétaux. Sans prétendre apporter de réponses définitives, l'auteur souhaite plutôt circonscrire des paradoxes: être en alliance sans renier sa fonction, accepter les différences sans pour autant 
adhérer au relativisme radical où tout se vaut, établir un dialogue tout en pouvant être critique de l'autre, travailler à l'unité d'une communauté sans fermer les yeux sur ses divergences internes... Le cas de la communauté sourde est certainement exemplaire pour illustrer les exigences de rencontre entre des identités lorsque l'on est intervenant ou chercheur.

L'organisation communautaire contemporaine est également traversée et influencée par les mises à jour théoriques des sciences sociales. On se rappelle que le rationalisme économique en action collective a connu ses heures de gloire, depuis les premières formulations de la théorie de la mobilisation des ressources dans les années 1960 et 1970. Pour simplifier, celle-ci réduit pratiquement l'action collective à une décision fondée sur la comparaison entre les coûts de la participation et les bénéfices escomptés (les acteurs décideront s'ils investissent du temps et combien, considérant la valeur du but poursuivi et leurs chances de l'atteindre). Or, dans son article, Martine Duperré souligne la place des émotions dans l'action collective à partir d'une recherche mettant à contribution différentes informations (notes d'observation, entrevues et documents) et portant sur plusieurs dizaines d'années d'existence de deux regroupements en santé mentale. On découvre que les émotions représentent des médiateurs dans toutes les étapes de l'action collective. Les valeurs, l'indignation, les attitudes, les goûts personnels, les tensions dans l'organisation et à l'égard d'autres acteurs, l'humour et l'amitié sont quelques-unes des manifestations des émotions dans l'action collective et dont l'organisation communautaire ne peut faire l'économie.

Un dernier aspect qui concerne l'organisation communautaire que rappelle Michelle Duval dans son article, est l'importance de la philosophie politique. En cela, bien des philosophes ont inspiré l'organisation communautaire et à chaque époque, on redécouvre tel ou tel auteur, sans doute parce que leur pensée permet d'expliquer et de donner un sens à ce qui se passe dans la société. L'auteure retient la contribution d'une femme ayant laissé sa marque dans les sciences sociales, Hannah Arendt (1906-1975), et pour cause. Cette philosophe politique développe sa pensée à partir des années 1950. Elle s'intéresse au totalitarisme et le dénonce, car il justifie le mal au nom d'un principe érigé au statut de vérité. Différents totalitarismes ont eu lieu, dont le nazisme qu'elle a fui. Le totalitarisme qui se développe dans la société moderne, soutient-elle, est l'individualisme. Pour Arendt, alors que le désespoir et la désolation ne touchent que des expériences marginales de la vie, ils représentent l'expérience usuelle de la majorité de la population dans la société moderne. Or, en plus du travail et de l'activité créative, l'action politique fait partie de la condition intrinsèque des humains agissant sur le monde. C'est pourquoi Arendt veut réhabiliter le politique comme espace public. Pour cela, il importe de mettre à contribution la capacité des humains de vouloir et de juger pour développer ces espaces publics. La démarche de la philosophe dans toute son œuvre est apparentée à l'art du questionnement, comme le pratiquait Socrate, et peut s'avérer inspirante sur le plan de la pratique pédagogique en organisation communautaire, comme le démontre Michelle Duval.

Évidemment, un numéro d'une revue ne peut prétendre rendre compte, à lui seul, de la complexité de l'organisation communautaire actuelle, ne serait-ce qu'en se limitant au 
Québec. C'est pourquoi la direction de la revue a fait appel à des lecteurs ayant bien voulu jeter un regard attentif à quatre ouvrages parus récemment sur l'organisation communautaire.

\section{RÉFÉRENCES BIBLIOGRAPHIQUES}

Ampleman, Gisèle, Gérald Doré, Lorraine Gaudreau, Claude Larose, Louise Leboeuf, et Denise Ventelou (1983). Pratiques de conscientisation, Montréal, Nouvelle Optique, 309 p.

AmPleman, Gisèle, Jocelyne Barnabé, Yvan Comeau, Gérald DorÉ, Ronald DuHAme, Lorraine Gaudreau, Colette Humbert, Jacques Lacroix, Louise LeboeUf, et Michel MatTe (1987). Pratiques de conscientisation 2, Québec, Collectif d'édition populaire, $366 \mathrm{p}$.

CASTELls, Manuel (2000). The Information Age: Economy, Society and Culture. Volume 2. The Power of Identity, Oxford, Blackwell Publishers, $461 \mathrm{p}$.

Lamoureux, Henri, Robert MAYER, et Jean PANET-RAYMOND (1984). L'intervention communautaire, Montréal, Éditions Saint-Martin, 237 p.

MARCOTTE, François (1986). L'action communautaire. Ses méthodes, ses outils, ses rouages et sa gestion, Montréal, Éditions Saint-Martin, 141 p.

SAVARIA, Marc (1985). Au ras des pâquerettes. Méthodes d'organisation communautaire, Drummondville, Éditions Luttes urbaines, $190 \mathrm{p}$. 